\title{
Sequence Components Obtained by Current Spectral Analysis in DC Motor Drives Working under Abnormal Conditions
}

\author{
G. Alonso Orcajo, J. M. Cano R., C. H. Rojas G., M. G. Melero, M. F. Cabanas \\ Dpto. de Ingeniería Eléctrica, Electrónica, de C. y S., \\ Edificio departamental $\mathrm{n}^{\circ} 4$, Campus de Viesques s/n. University of Oviedo \\ 33204. Gijón ASTURIAS. gonzalo@correo.uniovi.es
}

\begin{abstract}
This paper presents some results about the line current spectral analysis in a DC motor drive under abnormal working conditions. The dependency of the relative frequency of a harmonic and its sequence (positive/negative) is illustrated. Under some of the abnormal conditions noncharacteristic harmonics result. The frequency or sequence of these harmonics are different that the frequency components obtained under normal conditions. It is shown the result of the spectral analysis in four cases: a) under unbalanced supply voltage b) under distorted supply voltage c) under mechanical unbalanced of the DC motor d) under shortcircuit in the armature of the DC motor
\end{abstract}

Keywords. Harmonics, sequence components, adjustable speed drives

\section{Introduction}

Current spectral analysis is a power tool that has been used successfully in the prediction of the power systems working conditions.

Under normal conditions, the supply currents of a DC motor drive are influenced by the impedances of the ac and $\mathrm{dc}$ systems, the supply voltage, the mechanical load and the motor speed. It is said that the working point is normal when the motor is not damaged, the mechanical load is not variable and the supply voltage is free of distortion and unbalance. In previous papers have been published how the phase and amplitude of the supply current harmonics is modified when the working point of the drive is changed, but the frequency components are the same: positive components of the order $\mathbf{6 k + 1}$ and negative components of the order $6 \mathbf{k}-1$.

Under abnormal working conditions of the drive, new frequency lines and new sequence components are incorporated in the spectra of voltage and currents. In order to achieve the detection of the faults in the drive working conditions, the observation of the harmonic components is proposed as a good method Taking into account that DC drives are frequently operating in vital points of industrial plants, an efficient monitoring for early failure detection is becoming more and more important.
The analysis platform is constituted by:

- $5 \mathrm{kVA}$, six-pulse controlled rectifier

- Tachometer

- $4 \mathrm{~kW}$ DC motor

- $10 \mathrm{kVA}$, three-phase and programmable supply voltage

- Electrical brake to permit motor operation under different load levels

- Power analyzer for measurement of three phase voltages and line currents. It is capable of harmonic measuring.

- Coupling pieces to permit motor operation under different mechanical unbalance

- $\mathrm{AC}$ inductors to permit drive operation under different filtering conditions

- Personal computer to select the working point of the supply voltage

These experimental platform allow to study the evolution of the current spectra under different conditions: a) load levels, b) supply voltage, c) mechanical unbalance d) shortcircuit in the armature of the DC motor.

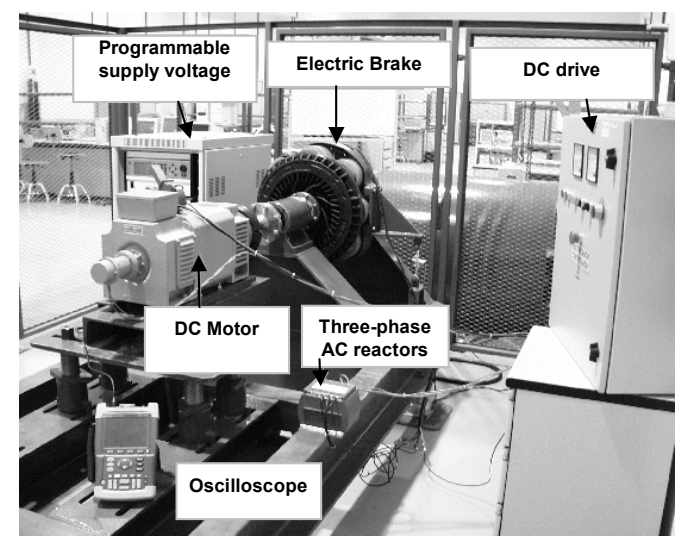

Fig. 1. Experimental platform

In order to study the evolution of the sequence components of the current spectra, a series of experimental tests were carried out under different degrees of abnormal conditions. This paper presents the experimental results obtained in such tests 


\section{Experimental analysis under unbalanced supply conditions}

In this section the behaviour of the current spectrum obtained under different unbalanced supply conditions will be shown.

In order to study the evolution of the sequence components of the current spectrum a series of experimental tests were carried out. In such tests the drive was supplied under different degrees of voltage unbalance. The current spectrum was analysed for different unbalance levels.

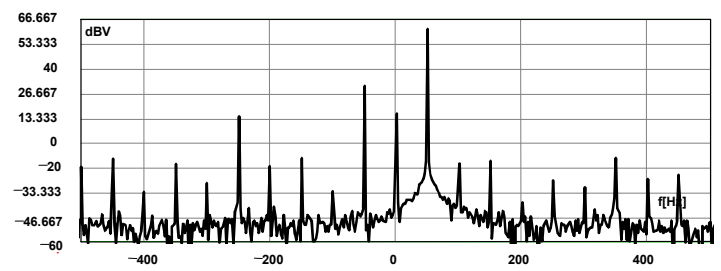

Fig. 2. Sequence components in the voltage spectrum

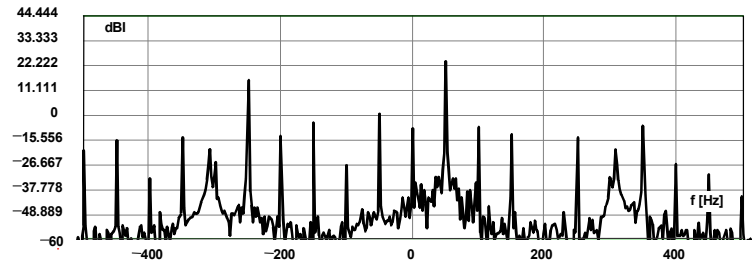

Fig. 3. Sequence components in the current spectrum

The evolution of the higher current harmonics under the presence of unbalanced voltage have been analysed. In order to illustrate the current spectrum evolution the results have been normalized and graphically presented.

The presence of high unbalance in the supply voltage particularly increases the negative component of the fundamental harmonic and the negative and positive component of the tripplen harmonics. Significant increases appear in the negative sequence component to harmonic frequencies $(6 \mathrm{k}+1) \mathrm{f}_{1}$ and in the positive sequence to harmonic frequencies $(6 \mathrm{k}-1) \mathrm{f}_{1}$.

\section{A. Evolution of the positive sequence components}

The following figure shows the evolution of the higher harmonics under the presence of unbalance voltage. Amplitudes have been normalized to the rms value of the fundamental component.

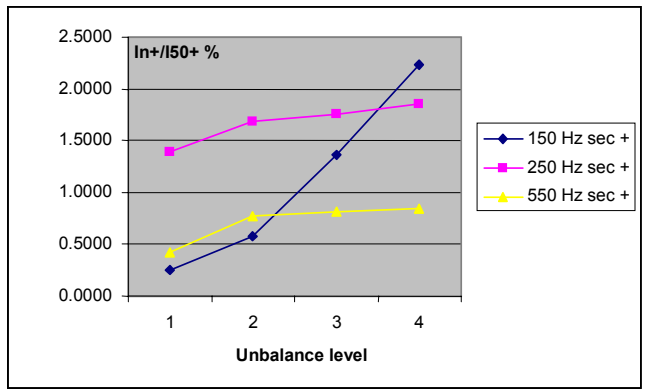

Fig. 4. Positive component amplitudes with constant load and different unbalance.

\section{B. Evolution of the negative sequence components}

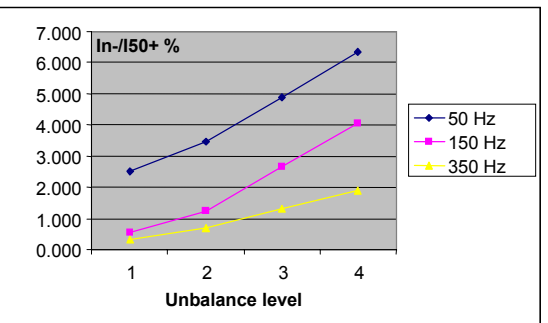

Fig. 5. Negative component amplitudes with constant load and different unbalance

\section{Experimental results obtained under distorted supply conditions}

In this section is analysed the effect of input voltage distortion on the drive input current. Some time-registers and line current spectra obtained under different levels of voltage distortion are shown. During the analysis the mechanical load and the motor speed keep constant.

In order to illustrate the current spectrum evolution some examples are considered. In the example 1 , the effect of a dominant harmonic voltage on the ac-side with frequency around $100 \mathrm{~Hz}$ is analysed. In the example 2, a fifth harmonic is injected into the ac bus.

The theory of harmonic transfer for dc-side to ac-side and vice-versa through a three-phase bridge converter is wellknown and provides powerful analysis techniques [2], [3].

\section{A. Example 1}

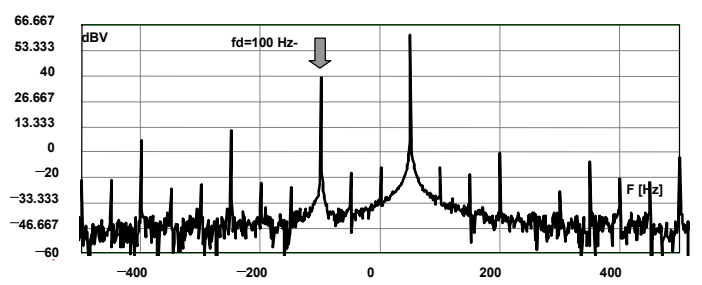

Fig. 6. Sequence components in the voltage spectrum Individual distortion Percentage of fundamental: 7,8 \%

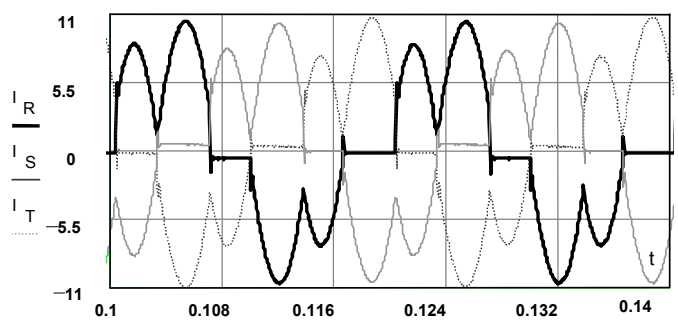

Fig. 7. Time-register of the supply currents

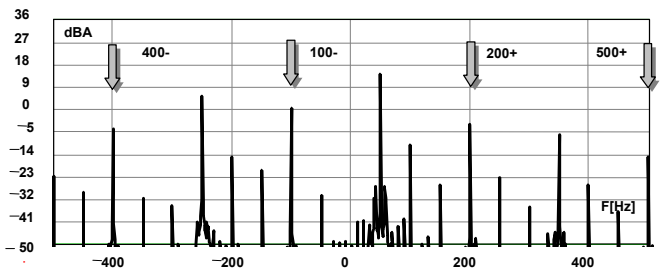

Fig. 8. Sequence components in the current spectrum 
Experimental analysis confirm the transfer mechanism of the harmonics. The amplitude of the harmonics with frequency $100 \mathrm{~Hz}, 200 \mathrm{~Hz}, 400 \mathrm{~Hz}$ and $500 \mathrm{~Hz}$ is increased when the individual distortion of the supply voltage have been increased.

\section{B. Example 2}

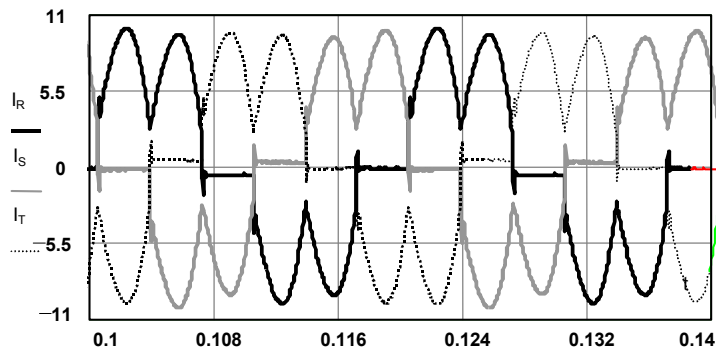

Fig. 9. Time register of the supply currents

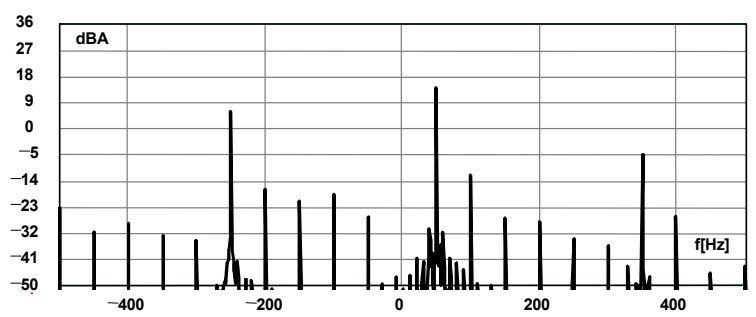

Fig. 10. Sequence components in the current spectrum

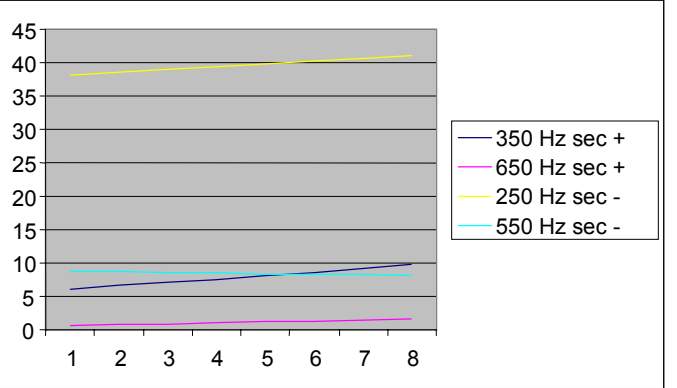

Fig. 11. Slight increase of the current harmonics amplitude

A fifth harmonic of negative sequence transferred to the dc side gives a $300 \mathrm{~Hz}$ component. This harmonic is obtained under ideal supply conditions. The current spectrum does not include new frequency lines and the amplitude variations of the harmonics are not significant.

\section{Experimental results obtained under mechanical unbalance of the de motor}

In this section the behaviour of a dc motor working under mechanical unbalance conditions is studied, and the possibility of diagnosing such failure by means of current analysis is considered. Under these conditions the evolution of the supply current harmonics in the dc drive and in the dc motor have been analysed.

An inertia wheel was coupled to the motor shaft. This wheel consist of some pieces that can modify its mass distribution and include different mechanical unbalance.
The current spectrums shown the presence of dc-side harmonic components multiples of the rotating speed and to the ac-side the corresponding two side-bands. The amplitude of these components are influenced by the mechanical unbalance degree.

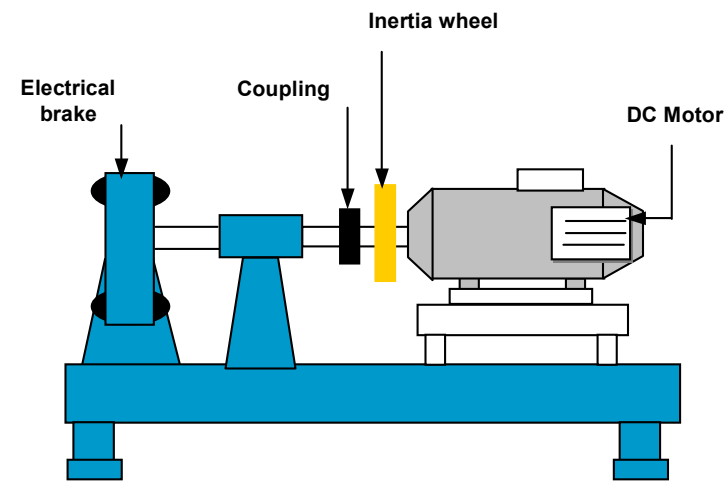

Fig. 12. Scheme of the test bed

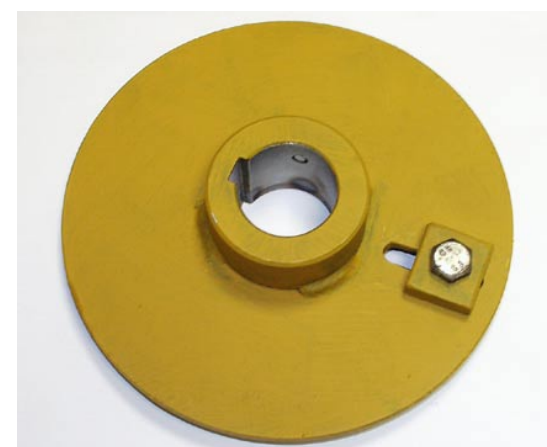

Fig. 13. Inertia wheel and coupling pieces.

The presence of unbalance in the motor will lead to an increase in the amplitude of the rotating speed harmonic and to a lesser extent to multiples of it. The diagnosis method consists of monitoring two harmonics and either side of the fundamental harmonic separated from it by $-\mathrm{f}_{\mathrm{r}}$ and $f_{r}$ where $f_{r}$ is the rotating frequency. The amplitudes of these two harmonics are related to the degree of unbalance that the motor has. The amplitude of all the current harmonics is a function of the load applied to the motor, so it is impossible to define an amplitude for sidebands that shows the limit between a balanced motor and one with unbalance. There is a series of frequencies besides these two harmonics that will also be related to unbalance of the motor. These side bands can be obtained for different values of the index $\boldsymbol{i}$ and for the frequency $\boldsymbol{i}_{\boldsymbol{i}} \boldsymbol{f}_{r}$ Only some of these harmonics will be appreciable in the drive currents.

This section will briefly introduce some conclusions that have been drawn as regards motor unbalance diagnosis using current spectrum analysis. Current spectrums from motors that have undergone laboratory analysis will be presented, clearly demonstrating the validity of sideband analysis around $50 \mathrm{~Hz}$.

Figure 14 shows the current spectrum of a de drive working under mechanical unbalance conditions when the rotating speed is 900 r.p.m $\left(\mathrm{f}_{\mathrm{r}}=900 / 60=15 \mathrm{~Hz}\right)$. The lower sideband has a 
frequency of $50-15=35 \mathrm{~Hz}$ and the higher sideband has a frequency of $50+15=65 \mathrm{~Hz}$.

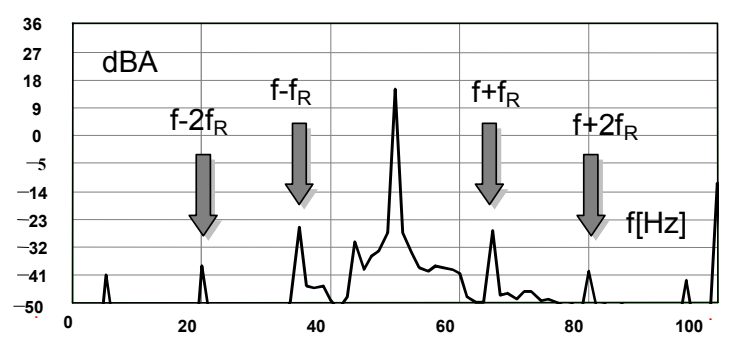

Fig. 14. Sequence components in the current spectrum

Current spectrums show two side bands close to the fundamental frequency. The rotating speed will affect frequencies induced in the supply currents. Figures 15 y 16 shows how amplitudes from the sideband harmonics increase as unbalance increases. During analysis the mechanical load and the motor speed remained constant.

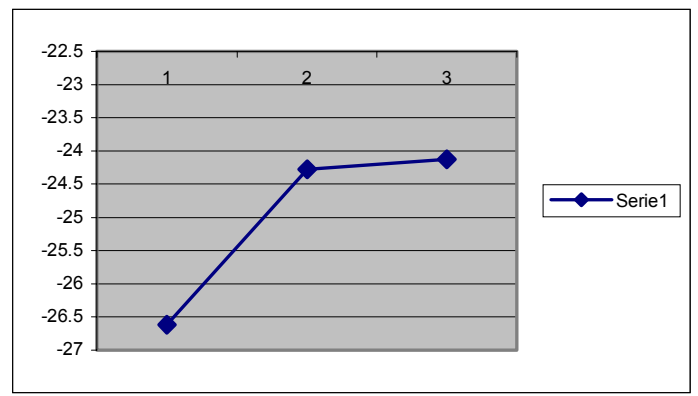

Fig. 15. Amplitude evolution of the harmonic with frequency $f+f_{r}$

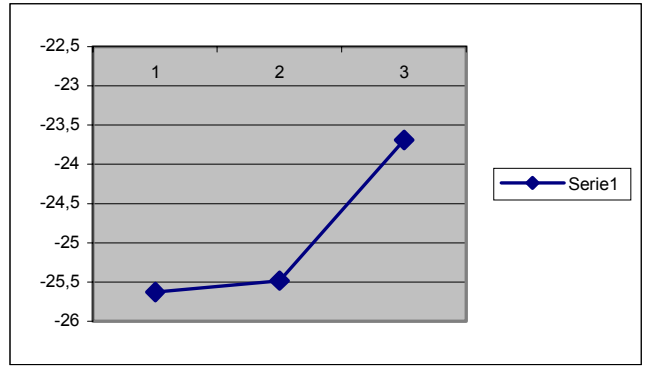

Fig. 16. Amplitude evolution of the harmonic with frequency $f-f_{r}$

\section{Experimental results obtained under shortcircuits in the armature of the motor}

It has been proven that when shortcircuits are presented in the armature of the motor, the current flowing through the armature increases. Two cases are shown. In the first case two adjacent conmutator segments are shortcircuited. In the second one four segments are shortcircuited.

The current spectrums show the presence of two side bands close to the fundamental frequency. The amplitudes of these two harmonics are related to the degree of shortcircuit that the armature experiences. It should be noted that the rotating speed and the number of shortcircuited segments will affect the frequencies induced in the supply currents.

\section{A. Two adjacent conmutator segments are short-circuited}

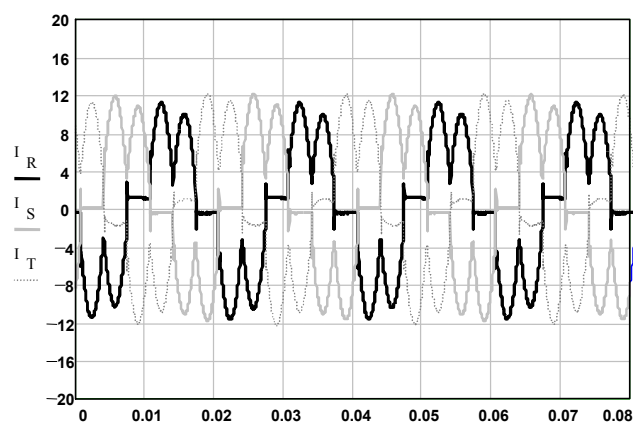

Fig. 17. Supply currents

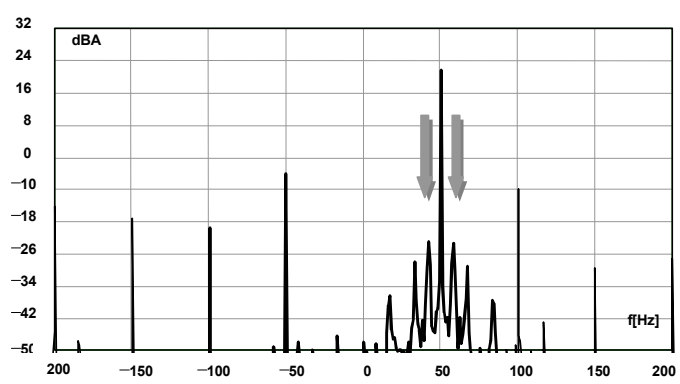

Fig. 18. Sequence components in the current spectrum

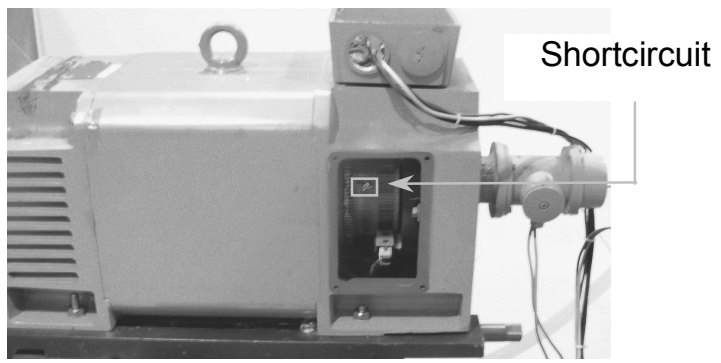

Fig. 19. Shortcircuit in the armature of the motor

\section{B. Four adjacent conmutator segments are short-circuited}

In this case, the short-circuit is severe damage. During analysis the mechanical load was very low and the motor speed remained constant.

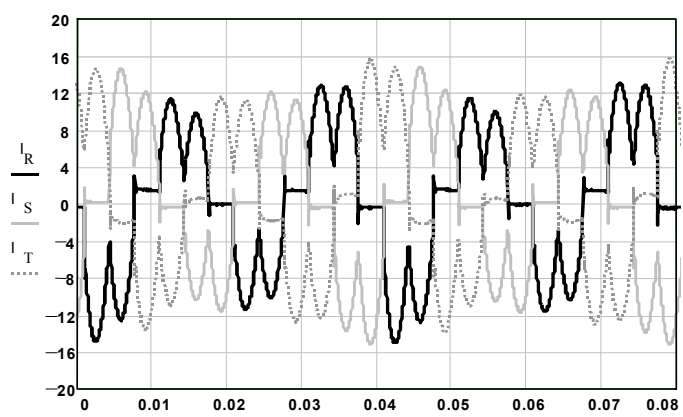

Fig. 20. Supply currents

Current spectrums show two side bands close to the fundamental frequency. The rotating speed will affect frequencies induced in the supply currents. 


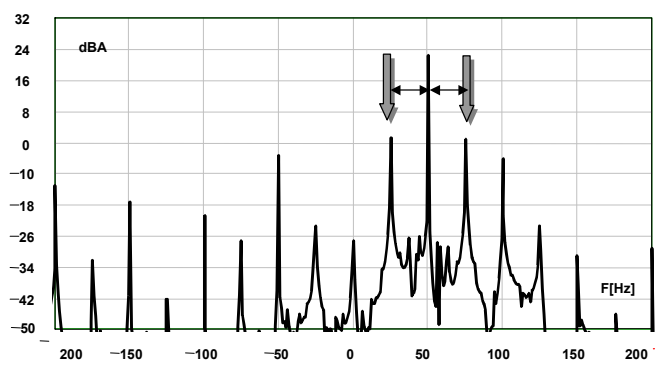

Fig. 21. Sequence components in the current spectrum

\section{Conclusions}

Practical criteria for applying current spectrum analysis to diagnosing abnormal working conditions in running drives $\mathrm{dc}$ motors have been presented. Some practical laboratoryproduced results have been shown. The abnormal working conditions that have been considered are: a) unbalanced supply voltage b) distorted supply voltage c) mechanical unbalanced of the DC motor d) shortcircuit in the armature of the DC motor.

\section{Acknowledgement}

The authors gratefully acknowledge Trefilería Moreda S.A. and the regional plan of Research and Development FICYT for their technical and financial support during this study.

\section{References}

[1] J. Arrillaga, B. C. Smith, N. R. Watson, A. R. Wood "Power System harmonic Analysis", Ed. John Willey \& Sons 1997.

[2] Lihua Hu, Robert Yacamini "Harmonic transfer through converters and HVDC links", IEEE Transactions on Power Electronics, Vol 7., N 3 July 1992

[3] Y. Jiang, A. Ekström "General analysis of harmonic transfer through converters", IEEE Transactions on Power Electronics, Vol 12., № 2 March 1997.

[4] R. Yacamini, J.W. Resende "Harmonic generation by HVDC schemes involving converters and static VAr compensators", IEE Proc. Gener. Transm Distrib., Vol 143, N 1 January 1996.

[5] Manfred Grötzbach, Michael Bauta "Significance of working point determining line current harmonics in controlled AC/DC converter" Proceedings Commercial Power Systems Technical Conference. Louisiana May 1996. 